\title{
Overview of the COMET Phase-I experiment
}

\author{
M. L. Wong ${ }^{* \dagger}$ \\ Osaka University \\ E-mail: m-wong@kuno-g.phys.sci.osaka-u.ac.jp
}

Charged Lepton Flavour Violation (CLFV) has yet to be observed experimentally. If observed, it would be a clear signal of new physics. One of the ways of observing this phenomena is via the neutrino-less conversion of muons to electrons bound to an atomic 1s ground state, $\mu^{-} N \rightarrow e^{-} N$ with the COMET experiment. This experiment is designed to look for $104.8 \mathrm{MeV}$ electrons converted from muons with a sensitivity a factor of 10000 better than that of current experimental limit [2]. From the J-PARC Main Ring, a dedicated $8 \mathrm{GeV}$ proton beam will be extracted into the J-PARC hadron hall. Upon its completion, COMET will use the world's most intense muon beam of the order of $10^{16}$ over 110 days capable of an experimental single-event sensitivity of $3 \times 10^{-17}$ or better. COMET Phase-I, will be using the Cylindrical Drift Chamber (CDC) as it is designed to avoid the high-radiation and high-hit rate due to the muon beam, muon decay-in-orbit (DIO) background events and low energy protons emitted by the muon nuclear capture process. Estimated backgrounds of the order of 0.02 events are sufficiently small for COMET Phase-I sensitivity of $10^{-15}$. The COMET collaboration has been advancing areas critical to the eventual deployment of the Phase-I since the beginning of 2011 with studies being done in muon yield simulations, trigger studies, data acquisition and so on.

Flavor Physics \& CP Violation 2015, May 25-29, 2015 Nagoya, Japan

* Speaker.

${ }^{\dagger}$ On behalf of the COMET Collaboration 

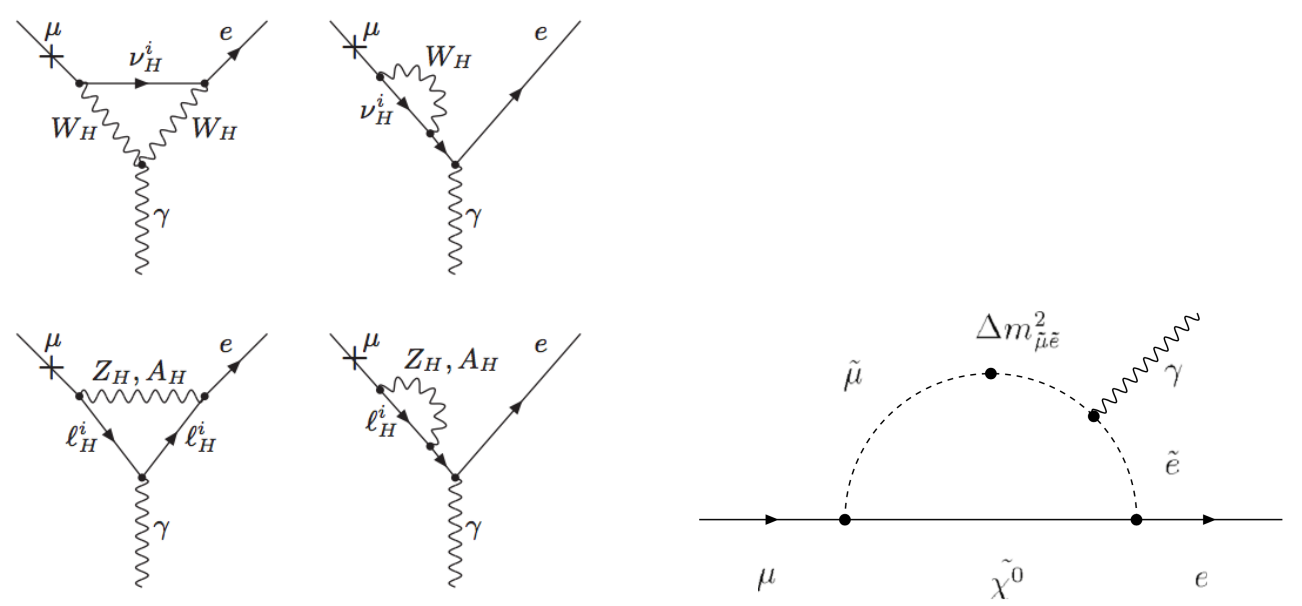

Figure 1: (Left) Littlest Higgs model with T-Parity diagrams contributing to the $\mu \rightarrow e$ process. H-subscript ed particles represent the heavy 'partners' of their SM particles. (Right) A SUSY loop diagram for a $\mu \rightarrow e$ process with the $\gamma$ possibly captured by the nucleus. $\Delta m_{\tilde{\mu} \tilde{e}}^{2}$ represents the magnitude of the off-diagonal matrix element for slepton mixing [3].

\section{Introduction}

The purpose of the COherent Muon to Electron Transition (COMET) experiment is to discover physics Beyond the Standard Model (BSM) via $\mu^{-} e^{-}$conversion. It is an effort to observe the conversion of muon bound to an atomic $1 \mathrm{~s}$ ground state of an aluminium atom. The muon in the $1 \mathrm{~s}$ orbital lives for about $864 \mathrm{~ns}$ [5] before the it decays in orbit or gets captured by the nucleus.

$$
\mu^{-}+A l \rightarrow e^{-}+A l^{*}
$$

The process in Eq.1.1 is very suppressed with a branching ratio of $10^{-54}$ in the Standard Model (SM). A possible way for a muon to change into an electron is via neutrino oscillations and Wboson interactions. In some BSM theories, a branching ratio of $10^{-15}$ is suggested. BSM theories like the Littlest Higgs model and Supersymmetry (SUSY) are some examples. Littlest Higgs [1] and SUSY feynman diagrams are shown in Fig.1.

\section{Experimental method and event signature}

COMET Phase-I consists of a curved solenoid connecting the pion production target and the detector section that houses the CyDet as shown in Fig.2. A pulsed proton beam power of $\sim 8 \mathrm{~kW}$ is directed at a graphite target to product short-lived pions (26ns). They travel along the curved superconducting solenoid but quickly decay into $\mu^{-}$and $\bar{v}_{\mu}$. The magnetic field selects only $\mu^{-}$ and while travelling they get momentum selected due to the curved trajectory. It hits the Al target inside the CyDet, creating muonic atoms. The $\mu^{-}$in the $1 \mathrm{~s}$-shell converts into an $e^{-}$, gains energy and leaves the $\mathrm{Al}$ atom. If there is a hit in the trigger hodoscope, the $\mathrm{CDC}$ records signal during the timing window as shown in Fig.3. 

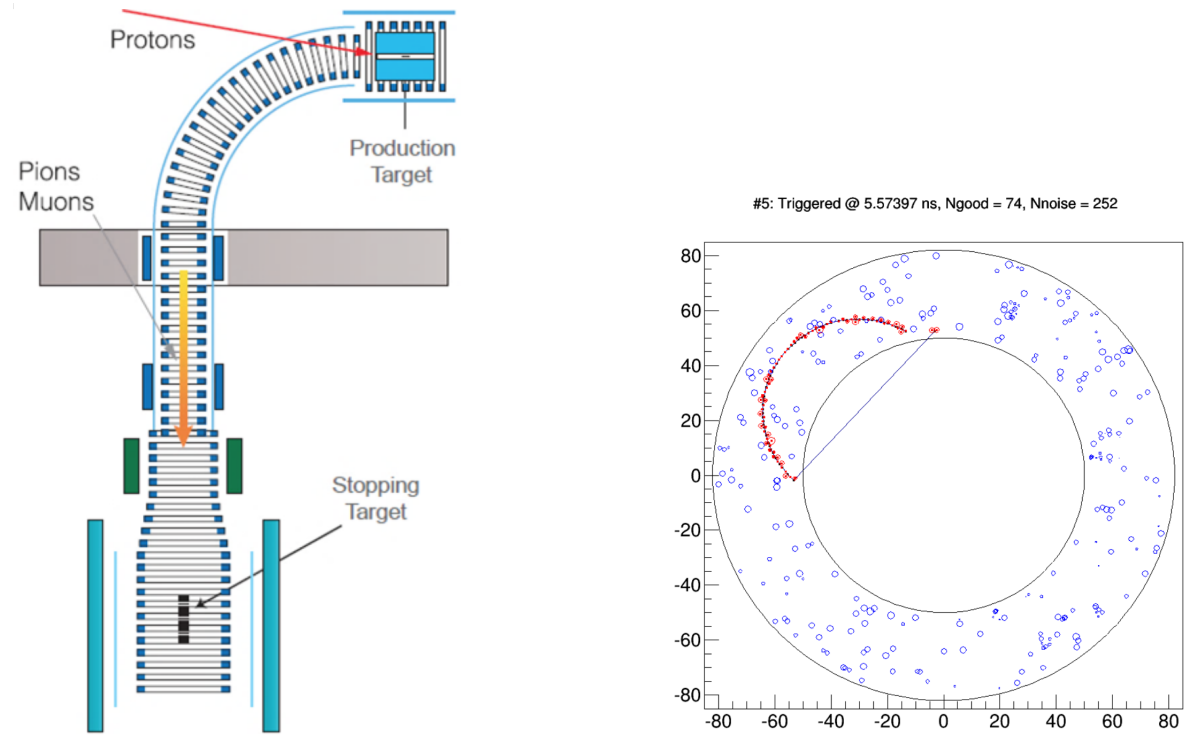

Figure 2: (Left) Schematic layout of COMET Phase-I. Goals of the first phase include measurement of proton beam extinction factors and other potential background sources to prepare for COMET Phase-II and to search for the $\mu^{-}-e^{-}$conversion with a SES of better than $3.1 \times 10^{-15}$. Beam characterization will be done by prototype sections of the Phase-II detectors. (Right) Simulation result of signal and accidental hit detector occupancy for the Cylindrical Detector (CyDet). The red dots are created by the converted electron that passed the energy deposit cut. The blue dots are noise hits that also passed the energy deposit cut. This event signature is from a mono-energetic single electron emitted from the conversion with an energy of $E_{\mu e}=m_{\mu}-B_{\mu}-E_{r e c}^{0} \sim 104.8 \mathrm{MeV}$.

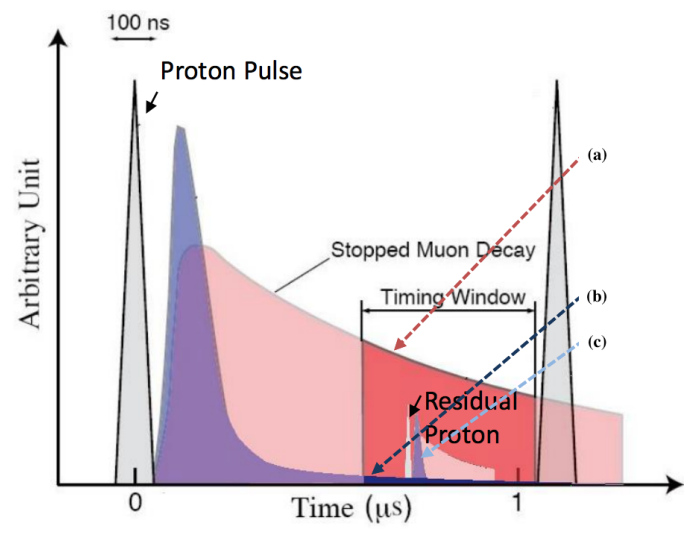

Figure 3: The timing window where measurement can be taken with an acceptable level of background. (a) Intrinsic physics backgrounds, (b) Beam related backgrounds (delayed), (c) Beam related backgrounds (prompt). 


\begin{tabular}{lr}
\hline Background & Estimated events \\
\hline Muon decay in orbit & 0.01 \\
Radiative muon capture & $5.6 \times 10^{-4}$ \\
Neutron emission after muon capture & $<0.001$ \\
Charged particle emission after muon capture & $<0.001$ \\
Beam electrons (prompt) & $8.3 \times 10^{-4}$ \\
Muon decay in flight (prompt) & $2.0 \times 10^{-4}$ \\
Pion decay in flight (prompt) & $\leq 2.3 \times 10^{-4}$ \\
Other beam particles (prompt) & $\leq 2.8 \times 10^{-4}$ \\
Radiative pion capture (prompt) & $\leq 2.3 \times 10^{-4}$ \\
Anti-proton induced backgrounds & 0.007 \\
Electrons from cosmic ray muons & $<0.0001$ \\
\hline Total & 0.019 \\
\hline
\end{tabular}

Table 1: Phase-I: Expected beam-related, intrinsic physics and cosmic ray backgrounds per event.

\section{Background sources and signal sensitivity}

DIO is a major contributor to background in the search for $\mu^{-} e^{-}$conversion in a muonic atom. Other background sources are listed in Table 1 [4]. Prompt background rates can be controlled with an efficient beam chopper and proton extinction system. The single event sensitivity (SES) is the experimental sensitivity to observe one event. It is defined by the number of muons stopping in the muon target, $N_{\mu}=2.0 \times 10^{18}$, the fraction of captured muons in $\mathrm{Al}, f_{\text {cap }}=0.6$, and the detector acceptance, $A_{e}=0.04$. For the CyDet,

$$
\begin{aligned}
\operatorname{SES}\left(\mu^{-}+A l \rightarrow e^{-}+A l^{*}\right) & =\frac{1}{N_{\mu} \cdot f_{\text {cap }} \cdot A_{e}} \\
& =3.1 \times 10^{-15}
\end{aligned}
$$

The upper limit is $7.2 \times 10^{-15}(90 \%$ C.L.) which is about 100 times better than the current published limit of $7 \times 10^{-13}$ (90\% C.L.) by SINDRUM-II at PSI [2].

\section{Current status and timeline}

COMET is organized in two phases. Phase-I studies the proton beam extinction and other potential background sources, in addition to searching for the $\mu \rightarrow e$ signal with the CyDet. Phase-II will be a much more sensitive version of the experiment. At present, wire stringing of the Cylindrical Detector (CDC) is in progress as shown in Fig.4. By mid 2016 the CDC should have been completed together with the DAQ electronics. By the end of 2016, CDC calibration and testing should be completed. In 2017, Phase-I data taking for 100 days and analysis. In the near future, COMET Phase-II will begin operation. It will add a $180^{\circ} \mathrm{C}$-shape bend to the muon transport solenoid and more advanced detectors such as a straw tracker and a electron calorimeter. This will improve the momentum resolution to reach the ultimate SES of $3 \times 10^{-17}$. 


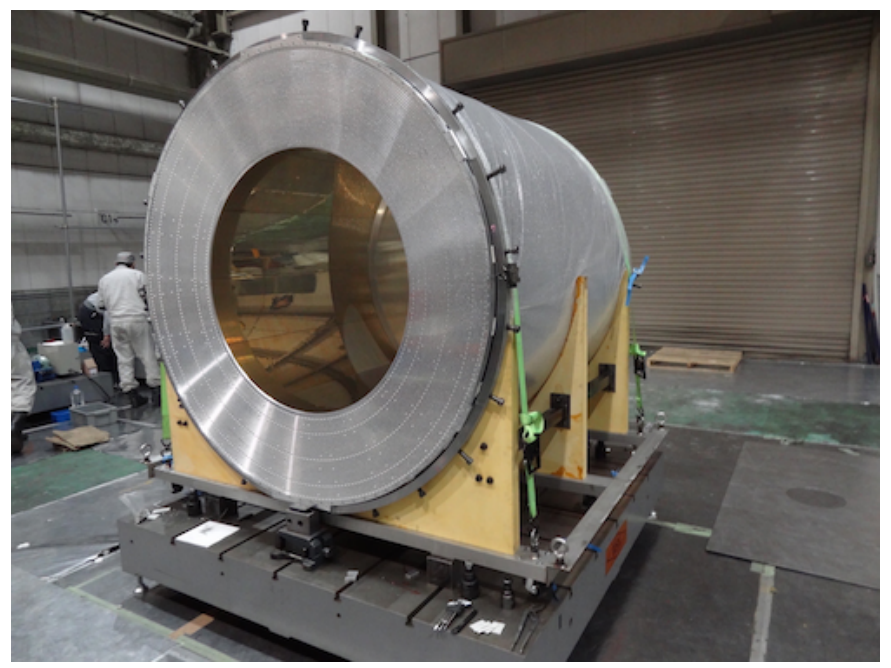

Figure 4: CDC being transported into the experimental hall.

\section{References}

[1] N. Arkani-Hamed, A. G. Cohen, E. Katz, and A. E. Nelson, The Littlest Higgs, JHEP 07 (2002), 034.

[2] W. Bertl, R. Engfer, E. A. Hermes, G. Kurz, T. Kozlowski, J. Kuth, G. Otter, F. Rosenbaum, N. M. Ryskulov, A. Van Der Schaaf, P. Wintz, and I. Zychor, A search for $\mu$-e conversion in muonic gold, European Physical Journal C 47 (2006), no. 2, 337-346.

[3] L. Calibbi, A. Faccia, A. Masiero, and S. K. Vempati, Lepton flavor violation from supersymmetric grand unified theories: Where do we stand for meg, prism/prime, and a super flavor factory, Phys. Rev. D 74 (2006), 116002.

[4] The COMET Collaboration, Comet technical design report, http: //comet.kek.jp/Documents_files/PAC-TDR-2014/PAC-Review-20141110.pdf, 2014.

[5] D. F. Measday, The nuclear physics of muon capture, Phys. Rept. 354 (2001), 243-409. 